\title{
Are postoperative prophylactic antibiotics in closed reduction of nasal bone fracture valuable?: prospective study of 30 cases
}

Nam Jang, Hyun Woo Shin

Department of Plastic and Reconstructive Surgery, Kangbuk Samsung Hospital, Sungkyunkwan University School of Medicine, Seoul, Korea
Background: Prophylactic antibiotic administration after surgery for a nasal bone fracture is performed due to concerns about infection-related complications, such as, toxic shock syndrome. To evaluate the validity and efficacy of antibiotic use, we compared the results obtained and the bacterial profiles of nasal packing materials in patients that underwent closed reduction for a nasal bone fracture with or without prophylactic antibiotic administration.

Methods: Thirty consecutive patients with a nasal bone fracture, but without an open wound, that underwent closed reduction during March to August 2017 were included in the present study. Fifteen of these 30 patients were randomly assigned to a control group, members of were administered postoperative intravenous antibiotics once at the day of surgery and then oral antibiotics for 4 days. The other 15 patients were assigned to an experimental group and not administered any antibiotic postoperatively. Antibiotic ointment was not applied to nasal packing in either group. Nasal packing was removed on postoperative day 4 in all cases. Removed nasal gauze packings were culture tested and strains identified in the two groups were compared.

Results: Bacterial strain types cultured from packings were similar in the experimental and control groups and no patient showed signs of clinically significant infection.

Conclusion: The findings of this study suggest postoperative prophylactic antibiotic use is not clinically required after closed reduction of a nasal bone fracture. Furthermore, the non-use of postoperative antibiotics is biologically beneficial, as it reduces the occurrence of resistant strains and medical costs, and is more convenient for patients.

Keywords: Anti-bacterial agent / Fractures, bone / Nasal bone / Therapeutics

\section{INTRODUCTION}

Nasal bone fracture is the most common fracture after facial trauma and is generally treated by closed reduction, which re-

Correspondence: Hyun Woo Shin

Department of Plastic and Reconstructive Surgery, Kangbuk Samsung Hospital,

Sungkyunkwan University School of Medicine, 29 Saemunan-ro, Jongno-gu,

Seoul 03181, Korea

E-mail: mdshin7@naver.com

This paper was presented at PRS Korea 2017 on November 11-12, 2017, in Seoul, Korea.

Received January 15, 2019 / Revised February 27, 2019 / Accepted March 5, 2019 quires a splint in the nasal cavity to stabilize the reduced bone fragment [1]. Foreign materials, such as, Vaseline gauze or Merocel (expandable hydroxylated polyvinyl acetate), are widely used as internal splints to maintain the reduced state. The need for postoperative prophylactic antibiotics after closed reduction is controversial. Despite the possibility of infection caused by nasal packing or nasal mucosa injury during the procedure $[2,3]$, no clear guideline has been issued regarding the preoperative or postoperative use of prophylactic antibiotics. Postoperative prophylactic antibiotic administration is performed due to concerns about infection-related complications, 
such as, toxic shock syndrome (TSS) [4] and acute sinusitis after nasal packing, although no information is available about the frequency of the latter. Despite low reported incidences of infections from nasal packing and a lack of published evidence on the efficacies of antibiotics for preventing such complications, postoperative prophylactic antibiotics are routinely prescribed.

The purpose of this prospective study was to compare the results of antibiotics use and bacterial profiles of nasal packing materials in 30 patients that underwent closed reduction of a nasal bone fracture and to evaluate the validity and efficacy of antibiotic use.

\section{METHODS}

The study was approved by the Institutional Review Board of Kangbuk Samsung Hospital (IRB No. 2018-09-032) and performed in accordance with the principles of the Declaration of Helsinki. In this prospective, randomized controlled study, 30 consecutive pure nasal bone fracture patients without an open wound that underwent closed reduction from March, 2017 to October, 2017 were included. Exclusion criteria were another facial bone fracture, a septal hematoma, a nasal mucosal injury, open comminuted fracture, chronic illness, such as, chronic renal failure or diabetes mellitus, and a history of recent antibiotic usage, chronic infectious sinusitis, or allergic rhinitis.

All operations were performed under general anesthesia. Patients received a single dose of preoperative prophylactic systemic intravenous antibiotic (cefazedone, a first-generation cephalosporin; $1 \mathrm{~g}$ ) to prevent intubation-related infection. Before nasal reduction, epinephrine-soaked gauzes were packed into both nostrils for 10 minutes to control epistaxis. After the fractured nasal bone and septum had been reduced using scalpel handle and Asch forceps, the nasal cavity was filled with a Vaseline gauze and Merocel to stabilize the reduced bone segment. An external nasal splint (Aqua splint) was also applied and maintained for 1 week after surgery.

Fifteen patients were allocated to group A, members of which received a single dose of postoperative systemic intravenous antibiotic (cefazedone, first-cephalosporin; $1 \mathrm{~g}$ ) at the day of sur-

Table 1. Study design

\begin{tabular}{lcc}
\hline & $\begin{array}{c}\text { Preoperative antibiotics } \\
\text { (at anesthesia induction) }^{\text {a) }}\end{array}$ & Postoperative antibiotics $^{b)}$ \\
\hline Group A & 0 & 0 \\
Group B & 0 & $\mathrm{X}$ \\
\hline
\end{tabular}

a) Intravenous antibiotics cefazedone $1 \mathrm{~g}$ once a day; ${ }^{b} \mid$ ntravenous cefazedone $1 \mathrm{~g}$ once a day $\&$ cephalexin $1 \mathrm{~g}$ three times a day (for 4 days). gery followed by oral antibiotic (cephalexin, first-cephalosporin; $1 \mathrm{~g}$ three times a day) for 4 days from day of surgery. The remaining 15 patients were allocated to group $B$ and not administered any systemic antibiotic postoperatively. No local antibiotic ointment was applied to nasal packing in either group (Table 1). Nasal packing (Merocel and Vaseline gauze) was removed on postoperative day 4 for all 30 patients. Culture testing was performed on removed nasal gauze packings and group bacterial profiles were compared. Identified bacteria were classified as normal flora or potential pathogens of the nasal cavity, that is, Streptococcus pneumoniae, Haemophilus influenzae, Moraxella catarrhalis, Streptococcus pyogenes, Staphylococcus aureus, Pseudomonas aeruginosa, and anaerobic bacteria (Prevotella, Porphyromonas, Fusobacterium, and Peptostreptococcus spp.) [5].

After removing nasal packing and nasal swabbing for microbiology, all patients underwent a thorough clinical examination, including an intranasal inspection, to detect signs of infection (heating sensation, swelling, persistent pain, purulent nasal drainage, and septal abscess) on postoperative days 4,11 . And we checked vital sign for all patients to evaluate general signs of infection. The postoperative follow-up and evaluation plan is detailed in Table 2.

\section{RESULTS}

The bacterial profiles identified in the two groups are shown in Fig. 1. Multiple strains were cultured in three patients in both groups.

Group bacterial profiles were similar in the two groups (Fig. 1). Identified strains were classified as potential pathogens or normal flora according to the criteria mentioned above [5]. The potential bacterial pathogens strains identified belonged to $S$. aureus and $P$. aeruginosa. Potential pathogen and S. aureus growth rates were not significantly different in the two groups (Table 3, Fig. 2).

During the study no clinical infection related to nasal packing occurred. No patient showed general signs of infection (fever and chill) or local signs of clinically significant infection (heating sensation, swelling, persistent pain, purulent nasal drainage, and septal abscess) on postoperative days 4, 11 in either group.

Table 2. Postoperative follow-up evaluation plan

\begin{tabular}{lcc}
\hline POD 4 & POD 11 & POD 30 \\
\hline Nasal packing removal & - & - \\
Packing gauze tip culture & - & - \\
Clinical examination & Clinical examination & Clinical examination \\
Intranasal inspection & Intranasal inspection & Intranasal inspection \\
\hline
\end{tabular}

POD, postoperative day. 


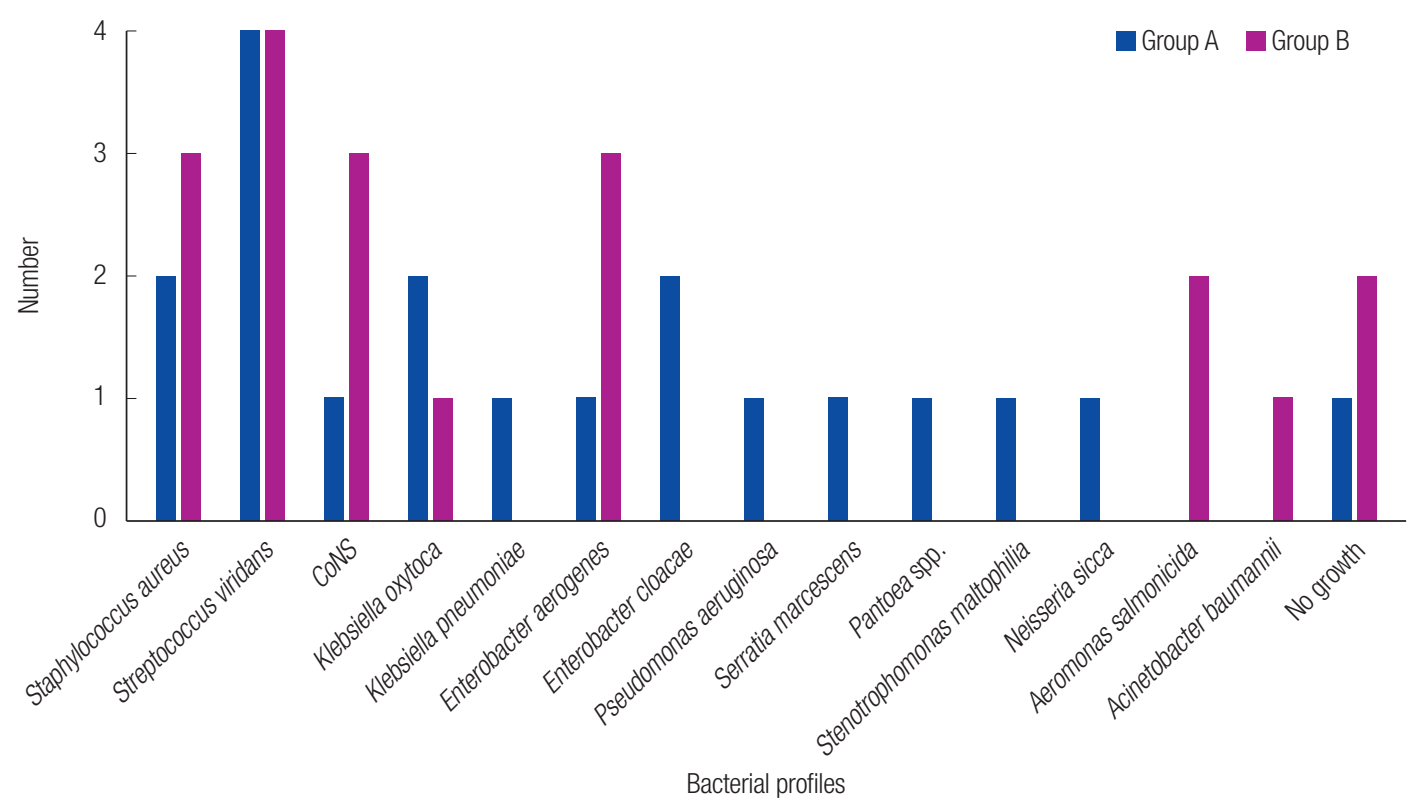

Fig. 1. Bacterial profiles identified in two groups. Multiple strains were cultured in each three patients in both groups. There were no significant differences in the bacterial profiles in these two groups of patients treated with or without postoperative prophylactic antibiotics. CoNS, coagulase-negative staphylococci.

Table 3. Potential pathogen and Staphylococcus aureus growth

\begin{tabular}{lcccc}
\hline & $\begin{array}{c}\text { Potential pathogen } \\
(\mathrm{n}=19)\end{array}$ & -value a $^{\mathrm{a}}$ & $\begin{array}{c}\text { S. aureus } \\
(\mathrm{n}=19)\end{array}$ & $p$-value \\
\hline Group A & 3 & 0.999 & 2 & 0.670 \\
Group B & 3 & & 3 & \\
\hline
\end{tabular}

a)Fisher exact test shows no significant differences in potential pathogen and $S$. aureus growth rate between the groups.

\section{DISCUSSION}

Nasal packing is used for a variety of reasons. Its common uses in the postoperative setting after nasal surgery include the control or prevention of epistaxis. After reducing nasal bone fractures, an internal vary splint is required to support reduced bone fragments and nasal packing provides the only means of internal splinting. Other roles of nasal packing include hemostasis, discharge absorption, and synechia prevention, and thus, it is considered an essential procedure after closed reduction [1], and concerns about infection-related complications due to the use of foreign materials for nasal packing has resulted in the widespread use of postoperative prophylactic antibiotics.

The method and optimal duration of nasal packing following closed reduction surgery for the correction of nasal bone fractures varies by surgeon. Intranasal packing is usually removed at 3-5 days after the operation. Following the study of Choi et al. [6] in 2015, the clinical outcome of nasal bone surgery is not altered by different duration of nasal packing. In this study, the author removed nasal packing on postoperative day 4 , which is

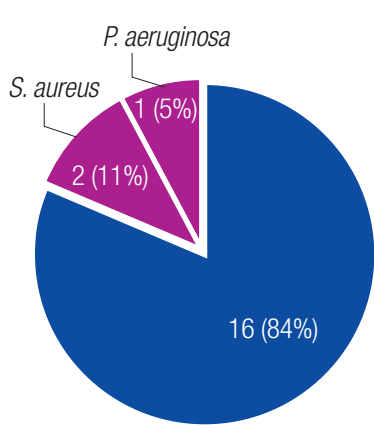

Group A normal flora $\quad$ Potential pathogen

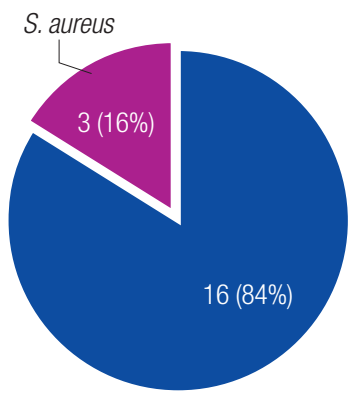

Group B
Fig. 2. Comparison of potential pathogen growth rate between two groups. When comparing the potential pathogen growth rate between two groups, there were same potential pathogen growth rate and no significant differences. S. aureus, Staphylococcus aureus; $P$. aeruginosa, Pseudomonas aeruginosa.

roughly average values of duration of nasal packing.

No validated guidelines have been issued on the use of postoperative prophylactic antibiotics after closed reduction of nasal bone fractures. Frequently, patients are started on postoperative prophylactic antibiotics after nasal packing due to fears of local infection and TSS, because accumulations of hemorrhagic fluids and secretions at surgical site causes packings to be converted into suitable media for bacterial growth. Furthermore, mucosal damage during surgery may play a role in the pathogenesis of TSS by enabling the transfer of bacteria to blood $[7,8]$. However, the incidence of TSS after nasal surgery has been reported to be $\sim 0.0002 \%$, which probably explains why antibiotic use has not been shown to decrease the incidence of TSS [9]. 
However, the prophylactic use of antibiotics can lead to significant adverse effects and costs. Unnecessary antimicrobial use inevitably increases risks of antimicrobial resistance [10], which is a growing problem throughout most of the developed world. Furthermore, penicillin-induced anaphylaxis contributes to 500-1,000 deaths per annum in the United States [11], and antibiotic-induced Clostridium difficile infections are responsible for $10 \%-25 \%$ of antibiotic-associated diarrhea cases [12] and have resulted in $\$ 4.8$ billion of excess cost in U.S. acute care facilities [13]. Thus, in view of the side effects and costs of systemic antibiotic use, the conventional use of postoperative prophylactic systemic antibiotics after closed reduction of nasal bone fractures requires careful consideration. The present study also shows the use of postoperative prophylactic systemic antibiotics after closed reduction of nasal bone fractures is probably unnecessary clinically and that it does not significantly alter bacterial profiles.

The present study has a number of limitations that warrant consideration. First, the sample size was too small to compare the distributions of cultured strains in the two groups, which precluded statistical analysis to support the need for postoperative prophylactic antibiotics. Second, we administered preoperative prophylactic systemic intravenous antibiotics to prevent intubation-related infections in both groups, and this may have inhibited postoperative nasal infections and biased our bacterial profile findings. Third, because the normal flora conditions are different for each patient, it will be more accurate to compare the pre-operative bacterial profiles and the bacterial profiles after antibiotic treatment in each patient, and future studies will reflect this. Following the study of Breda et al. [14] in 1987, preoperative toxic shock syndrome toxin-1 (TSST-1) producing $S$. aureus isolation was found to correlate well with development of postoperative TSS-like symptoms. So, additional toxin assay can be helpful in future studies. Nevertheless, this is the first comparative study on postoperative nasal bacterial profiles after closed reduction of nasal bone fractures. We hope the study is further developed by performing nasal cultures from larger numbers of patients and statistical analysis.

In the present study, the use of postoperative prophylactic antibiotics after closed reduction did not significantly alter bacterial profiles identified by nasal packing material culture or the clinically infection rate. These findings suggest postoperative prophylactic antibiotic use is not required after closed reduction of nasal bone fractures. Furthermore, the non-use of postoperative antibiotics is known to reduce the occurrences of resistant strains and obviously reduces costs and eliminates the risks associated with prophylactic antibiotics use.

\section{NOTES}

\section{Conflict of interest}

No potential conflict of interest relevant to this article was reported.

\section{Ethical approval}

The study was approved by the Institutional Review Board of Kangbuk Samsung Hospital (IRB No. 2018-09-032) and performed in accordance with the principles of the Declaration of Helsinki. Written informed consent was obtained.

\section{ORCID}

Nam Jang https://orcid.org/0000-0001-5155-7480

Hyun Woo Shin https://orcid.org/0000-0003-4396-3395

\section{REFERENCES}

1. Moon SH, Baek SO, Jung SN, Seo BF, Lee DC, Kwon H. Efficacy of biodegradable synthetic polyurethane foam for packing nasal bone fractures. J Craniofac Surg 2012;23:1848-50.

2. Titiz A, Zeyrek T, Ozcan M, Sabuncuoglu B, Yilmaz YF, Unal A. The effects of merocel and glove finger tampon applications on the nasal septum mucosa of rabbits. Rhinology 2008;46:112-5.

3. Shaw CL, Dymock RB, Cowin A, Wormald PJ. Effect of packing on nasal mucosa of sheep. J Laryngol Otol 2000;114:506-9.

4. Jacobson JA, Kasworm EM. Toxic shock syndrome after nasal surgery: case reports and analysis of risk factors. Arch Otolaryngol Head Neck Surg 1986;112:329-32.

5. Brook I. Microbiology of sinusitis. Proc Am Thorac Soc 2011; 8:90-100.

6. Choi DS, Lee JW, Yang JD, Chung HY, Cho BC, Choi KY. Minimal packing duration in close reduction for nasal bone fracture treatment. J Plast Surg Hand Surg 2015;49:275-9.

7. Todd JK, Todd BH, Franco-Buff A, Smith CM, Lawellin DW. Influence of focal growth conditions on the pathogenesis of toxic shock syndrome. J Infect Dis 1987;155:673-81.

8. Tierno PM Jr, Hanna BA. Magnesium and the production of toxic-shock-syndrome toxin-1 by Staphylococcus aureus. J Infect Dis 1986;153:994-6.

9. Jacobson JA, Stevens MH, Kasworm EM. Evaluation of singledose cefazolin prophylaxis for toxic shock syndrome. Arch Otolaryngol Head Neck Surg 1988;114:326-7.

10. Chisholm-Burns MA, Schwinghammer TL, Wells BG, Malone PM, Kolesar JM, Dipiro JT. Pharmacotherapy principles and practice. New York: McGraw-Hill; 2010.

11. Neugut AI, Ghatak AT, Miller RL. Anaphylaxis in the United States: an investigation into its epidemiology. Arch Intern Med 
2001;161:15-21.

12. Bartlett JG. Detection of Clostridium difficile infection. Infect Control Hosp Epidemiol 2010;31 Suppl 1:S35-7.

13. Dubberke ER, Olsen MA. Burden of Clostridium difficile on the healthcare system. Clin Infect Dis 2012;55 Suppl 2:S88-92.
14. Breda SD, Jacobs JB, Lebowitz AS, Tierno PM Jr. Toxic shock syndrome in nasal surgery: a physiochemical and microbiologic evaluation of Merocel and NuGauze nasal packing. Laryngoscope 1987;97:1388-91. 